\title{
Screening for Adverse Drug Events: a Randomized Trial of Automated Calls Coupled with Phone-Based Pharmacist Counseling
}

\author{
Gordon D. Schiff, $M D^{1,2}$, Elissa Klinger, $S M^{1,3}$, Alejandra Salazar, PharmD ${ }^{7}$, Jeffrey Medoff, $B A^{7}$, \\ Mary G. Amato, PharmD4, E. John Orav, $P D^{1,2}$, Shimon Shaykevich, $\mathrm{MS}^{7}$, \\ Enrique V. Seoane, PharmD ${ }^{5}$, Lake Walsh, $B A^{7}$, Theresa E. Fuller, $B A, B S^{1,6}$, \\ Patricia C. Dykes, RN, PhD, MA ${ }^{1,2}$, David W. Bates, MD, MSc ${ }^{1,2}$, and Jennifer S. Haas, MD, MSPH $H^{1,2}$
}

'Division of General Internal Medicine and Primary Care, Brigham and Women's Hospital, Boston, MA, USA; ${ }^{2}$ Harvard Medical School, Boston, MA, USA; ${ }^{3}$ Penn Medicine, University of Pennsylvania Health System, Philadelphia, PA, USA; ${ }^{4}$ Massachusetts College of Pharmacy and Health Sciences University, Boston, MA, USA; ${ }^{5}$ School of Pharmacy, Chapman University, Orange, CA, USA; ${ }^{6}$ Northeastern University, Boston, MA, USA.

BACKGROUND: Medication adverse events are important and common yet are often not identified by clinicians. We evaluated an automated telephone surveillance system coupled with transfer to a live pharmacist to screen potentially drug-related symptoms after newly starting medications for four common primary care conditions: hypertension, diabetes, depression, and insomnia.

METHODS: Cluster randomized trial with automated calls to eligible patients at 1 and 4 months after starting target drugs from intervention primary care clinics compared to propensity-matched patients from control clinics. Primary and secondary outcomes were physician documentation of any adverse effects associated with newly prescribed target medication, and whether the medication was discontinued and, if yes, whether the reason for stopping was an adverse effect.

RESULTS: Of 4876 eligible intervention clinic patients who were contacted using automated calls, 776 (15.1\%) responded and participated in the automated call. Based on positive symptom responses or request to speak to a pharmacist, 320 patients were transferred to the pharmacist and discussed 1021 potentially drug-related symptoms. Of these, 188 (18.5\%) were assessed as probably and $479(47.1 \%)$ as possibly related to the medication. Compared to a propensity-matched cohort of control clinic patients, intervention patients were significantly more likely to have adverse effects documented in the medical record by a physician (277 vs. 164 adverse effects, $p<0.0001$, and 177 vs. 122 patients discontinued with documented adverse effects, $p<0.0001$ ).

DISCUSSION: Systematic automated telephone outreach monitoring coupled with real-time phone referral to a pharmacist identified a substantial number of previously unidentified potentially drug-related symptoms, many of which were validated as probably or possibly related to the drug by the pharmacist or their physicians. Multiple

Electronic supplementary material The online version of this article (https://doi.org/10.1007/s11606-018-4672-7) contains supplementary material, which is available to authorized users.

Received February 7, 2018

Revised July 10, 2018

Accepted August 30, 2018

Published online October 5, 2018 challenges were encountered using the interactive voice response (IVR) automated calling system, suggesting that other approaches may need to be considered and evaluated.

TRIAL REGISTRATION: ClinicalTrials.gov: NCT02087293

J Gen Intern Med 34(2):285-92

DOI: $10.1007 / \mathrm{s} 11606-018-4672-7$

(c) Society of General Internal Medicine 2018

\section{INTRODUCTION}

Use of pharmacologic agents is ubiquitous, with more than half of the US population reporting using a prescription medication in the past year. ${ }^{1,2}$ Medication management dominates medical encounters, with two thirds of adult ambulatory care visits resulting in a prescription or continuation of a medication, and $25 \%$ noting a new or changed prescription. ${ }^{1}$ Prior work has demonstrated that up to one in four patients prescribed a new medication experiences an adverse drug event (ADE). ${ }^{3,4}$ The burden of outpatient ADEs on patients and health systems is substantial, resulting in more than 3.5 million physician office visits and one million US emergency room visits annually. ${ }^{5-8}$

Timely identification of symptoms related to ADEs is important to minimize harm and identify drug adverse effects more broadly. Unfortunately, many ADEs go undetected, unreported, and unaddressed..$^{9-11}$ While various methods educate patients about potential ADEs (e.g., leaflets or counseling at pharmacies) and clinicians often ask about potential ADEs during follow-up encounters, proactive approaches for monitoring and detecting $\mathrm{ADEs}$ are rare or nonexistent in most settings.

We have deployed a variety of approaches to monitor patients for symptoms associated with starting new medications. $^{3,} 12$ Given the large numbers of medications initiated in ambulatory care, manual efforts such as telephone calls should 
be replaced by more automated approaches to enable more efficient outreach to larger numbers of patients at risk. ${ }^{13}$ One approach is interactive voice response (IVR) technology, which enables automated telephone calls. ${ }^{14,}{ }^{15}$ IVR systems use various features including voice recognition and data capture; we have previously used IVR as a tool for pharmacoepidemiologic monitoring of ADEs. ${ }^{16-18}$

In this study, we deployed IVR to call patients newly prescribed medications for hypertension, diabetes, insomnia, and depression. We developed and implemented an IVR system to contact patients approximately 1 month and 4 months after receiving a new prescription for a medication for one of these four conditions. An additional feature of this proactive surveillance was real-time transfer to a live clinical pharmacist for patients reporting potential drug-related symptoms. Here, we describe the results of a trial evaluating the impact of this approach.

\section{METHODS}

\section{Overview}

Calling for Earlier Detection of Adverse Reactions (CEDAR) was a cluster randomized controlled trial of adult primary care patients receiving care at practices affiliated with Brigham and Women's Hospital and North Shore Physician's Group. Using expert review and frequency of use, we identified 112 medications used for the four target conditions. Patients newly started on target medications from intervention clinics were called using a novel IVR platform for detecting patientreported symptoms. Patients were transferred in real-time to a clinical pharmacist to assess whether the symptom was related to the new medication, filed a note in the electronic health record (EHR), and notified the appropriate clinician if symptoms required urgent follow-up. All clinics shared the same EHR, the Partners HealthCare (PHS) Longitudinal Medical Record (LMR). Clinics were randomized to intervention or control sites (or where possible, randomized by suites within clinics at each site). A cohort of propensity scorematched patients from control clinics was assembled to match patients who participated in the calls from intervention clinics. We called patients at 1 month to detect potential acute reactions and at 4 months to detect potential subacute problems. The research protocol was approved by the PHS Institutional Review Board and registered at Clinicaltrials.gov (NCT NCT02087293).

\section{IVR Script Development}

We developed an IVR survey based on prior work ${ }^{17-19}$ which included a consent script, birthdate verification, and set of generic symptom questions followed by selected drugspecific questions (e.g., cough for ACE inhibitors). Questions required either numeric (birthday) or dichotomous YES/NO answers, with "SKIP" possible. Patients could exit the survey at any time by hanging up. The IVR script was translated into Spanish and patients were asked by the IVR system whether they preferred to participate in English or Spanish. The IVR questionnaire logic was mapped in Visio and programmed by a commercial IVR vendor (Vocantas) (Supplemental Appendix).

\section{Patient Eligibility}

Twenty-six clinic sites were identified and matched based on underlying characteristics; 13 clinic units were randomized to the intervention group. Control clinics provided patient data for chart review but no patients or providers were contacted. We included all patients over age 18 who (1) received a prescription for one of the target medications at one of the 26 clinic units; (2) evidenced no history of having been prescribed the target medication within the previous 2 years; and (3) received a prescription for more than seven doses (e.g., excluded benzodiazepine prescriptions for medical procedures or air-flight). All English- or Spanish-speaking patients were included. If an eligible patient was prescribed more than one target medication, we randomly selected only one. Patients could be enrolled/recruited for the study only once.

\section{Randomization and Recruitment}

Clinics were stratified (based on size, demographic characteristics, and network), then were randomized to intervention or control sites. Patients in intervention clinics who met eligibility criteria were sent an informational mailing describing the study and provided a phone number to call if the patient wished to opt out from receiving the call. Two weeks later (i.e., 1 month after the prescription), intervention clinic patients who had not opted out received IVR calls. Patients were called between 9 am and $7 \mathrm{pm}$, on a rotating schedule based on availability of study staff to receive transferred calls. Patients received up to 10 calls over a 2-week period, including at least one weekend day. Once reached, patients could opt out, provide verbal consent, or hang up at any point during the call. Participants were asked to verify their identity (by birthdate).

A cohort of control patients who met the same eligibility criteria was generated from control sites using propensity score matching. A logistic regression model estimated the probability that a patient would participate on the telephone interview. The model used sex, age, race, ethnicity, religion, marital status, median household income, availability of email, presence of a PCP, number of primary care visits, target drug, and diagnoses of diabetes, hypertension, depression, and insomnia as predictors. This model was then applied to the control patients to calculate a propensity score, estimating each control clinic patient's probability of responding to the telephone survey had they been called. The propensity score of each intervention patient who responded to the telephone survey was then matched to the closest propensity score of a control patient; the pair of patients would then have an equal likelihood of responding to the survey. 


\section{Data Collection and Study Flow}

Following consent by IVR, intervention participants were queried about their adherence to the target medication, and asked generic and drug-specific adverse effect questions. Any patient reporting new symptoms since starting the medication was transferred to the pharmacist who reviewed their symptoms and assessed the likelihood of a significant adverse effect, as well as triaged the patient based on their need and urgency for additional medical attention. Patients who did not report symptoms on the IVR call were also offered an opportunity to speak to the live pharmacist for any medicationrelated questions.

\section{Pharmacist Protocol}

The pharmacist simultaneously received the transferred call and was notified electronically via email listing symptoms reported in the IVR survey. During the call, the pharmacist inquired about adherence to the prescription and then discussed each symptom with the patient and evaluated the severity (life-threatening, serious, significant, and mild), urgency (immediate attention needed, moderate, and non-urgent), and likelihood that the symptom was drug-related (definite, probable, possible, and unlikely) using previously published modified Naranjo algorithms. ${ }^{20}$ After the call, the pharmacist documented the intervention in the EHR. For patients with life-threatening and/or urgent ADEs, the patient's physician was contacted. The pharmacist spoke both English and Spanish and counseled patients in their preferred language.

\section{Outcome Measures}

The primary outcome was physician documentation of any adverse effects associated with the target newly prescribed medication. This was assessed by comprehensive manual chart review of 1 year of all notes following the target medication prescription by trained research assistants. Reviewers recorded all instances a physician note commented on a potential adverse event related to the target medication. Secondary outcomes included whether the study medication had been discontinued within this time and, if so, whether it was stopped due to potential adverse effects.

\section{Statistical Analysis}

Characteristics of intervention patients and their propensity-matched controls were compared using chisquare tests for categorical characteristics and $t$ tests or Wilcoxon rank sum tests for continuous measures (Table 1). The percentages of unique patients with symptoms documented in the two arms were compared using chi-square tests. The numbers of symptoms documented in each arm were compared using Poisson regression since individual patients could have multiple events. For documented symptoms due to the target medication, with the available sample size of 776 controls and a documentation rate of $15.5 \%$, we had $90 \%$ power if the documentation rate in the 776 intervention patients was $22 \%$ or higher. For medication discontinuation due to ADEs with available sample size of 5897 controls and discontinuation rate of $11 \%$, we had $90 \%$ power as long as discontinuation rate in 5106 intervention patients was $13 \%$ or higher.

Table 1 Baseline Characteristics of Intervention Participants and Matched Controls

\begin{tabular}{|c|c|c|c|}
\hline & $\begin{array}{l}\text { Intervention participants } \\
n=776\end{array}$ & $\begin{array}{l}\text { Matched control patients } \\
n=776\end{array}$ & $p$ value \\
\hline Age (mean/median (range)) & $57.2 / 59.0(18-96)$ & $59.7 / 60(19-97)$ & 0.0005 \\
\hline Race/ethnicity* & & & $<0.0001$ \\
\hline White, no. (\%) & $522(67.3)$ & $593(76.4)$ & \\
\hline Black, no. (\%) & $108(13.9)$ & $63(8.1)$ & \\
\hline Hispanic, no. (\%) & $112(14.4)$ & $79(10.2)$ & \\
\hline Other, no. $(\%)$ & $34(4.4)$ & $41(5.3)$ & \\
\hline Sex & & & 0.0005 \\
\hline Female, no. $(\%)$ & $518(66.6)$ & $581(74.9)$ & \\
\hline \multicolumn{4}{|l|}{ Clinical characteristics } \\
\hline Charlson Index (mean/median) & $1.5781 / 1.0000$ & $1.4867 / 1.0000$ & 0.6458 \\
\hline No. of medications ${ }^{\dagger}$ (mean/median) & $10.1 / 8.0$ & $10.2 / 8.0$ & 0.83 \\
\hline No. of medications at 1 year ${ }^{\dagger}$ (mean/median) & $11.5 / 10.0$ & $11.3 / 9.0$ & 0.71 \\
\hline No. of medical problems ${ }^{\dagger}$ (mean/median) & $9.1 / 8.0$ & $9.2 / 8.0$ & 0.75 \\
\hline No. of medical problems at 1 year $^{\dagger}$ (mean/median) & $10.2 / 9.0$ & $10.2 / 9.0$ & 0.92 \\
\hline Condition treated with target medication & & & $\begin{array}{l}\text { Overall target } \\
\text { condition } \\
\text { comparison } \\
0.3389\end{array}$ \\
\hline Hypertension, no. (\%) & $411(53.0)$ & $442(57.0)$ & 0.1134 \\
\hline Insomnia, no. $(\%)$ & $143(18.4)$ & $132(17.0)$ & 0.4701 \\
\hline Depression, no. (\%) & $122(15.7)$ & $120(15.5)$ & 0.9136 \\
\hline Diabetes, no. (\%) & $100(12.9)$ & $82(10.5)$ & 0.1415 \\
\hline
\end{tabular}

*Race/ethnicity data based on coded fields in the EHR

† Medications and documented problems extracted from the EHR medication and problem lists at the time the target medication was prescribed and 1 year following the target prescription date

tTreatment indication based on chart and not patient self-report 


\section{RESULTS}

\section{Recruitment}

Between June 2013 and April 2015, 5143 eligible patients were identified by EHR in intervention clinics and 5985 in non-intervention control clinics. IVR calls were placed to 4876 (94.8\%) eligible intervention patients, of whom 894 (17.4\%) gave initial phone consent and 776 (15.1\%) provided answers to at least one question in the automated survey and were considered participants. The 3982 intervention patients who did not participate included 540 (10.5\%) who were never reached, 2547 (49.5\%) who immediately hung up, and 895 $(17.4 \%)$ who declined to participate in the call (Fig. 1).

\section{Study Population}

Table 1 compares EHR-derived demographic characteristics and comorbidities of participants and matched controls. Compared to matched controls, participants were slightly younger (median age 59 vs. 60$)$, less likely female (66.8\% vs. $74.9 \%$, $p=0.0005$ ), and more racially/ethnically diverse. No significant differences were observed between intervention participants and controls with respect to Charlson comorbidity indices. We observed no significant differences in the number of active medications and problems documented in the EHR for the intervention vs. control groups, either at the time of the target medication prescription or after 1 year. The majority of the participants $(53.0 \%)$ were recruited for a target medication to treat hypertension; $18.4 \%$ were recruited for an insomnia

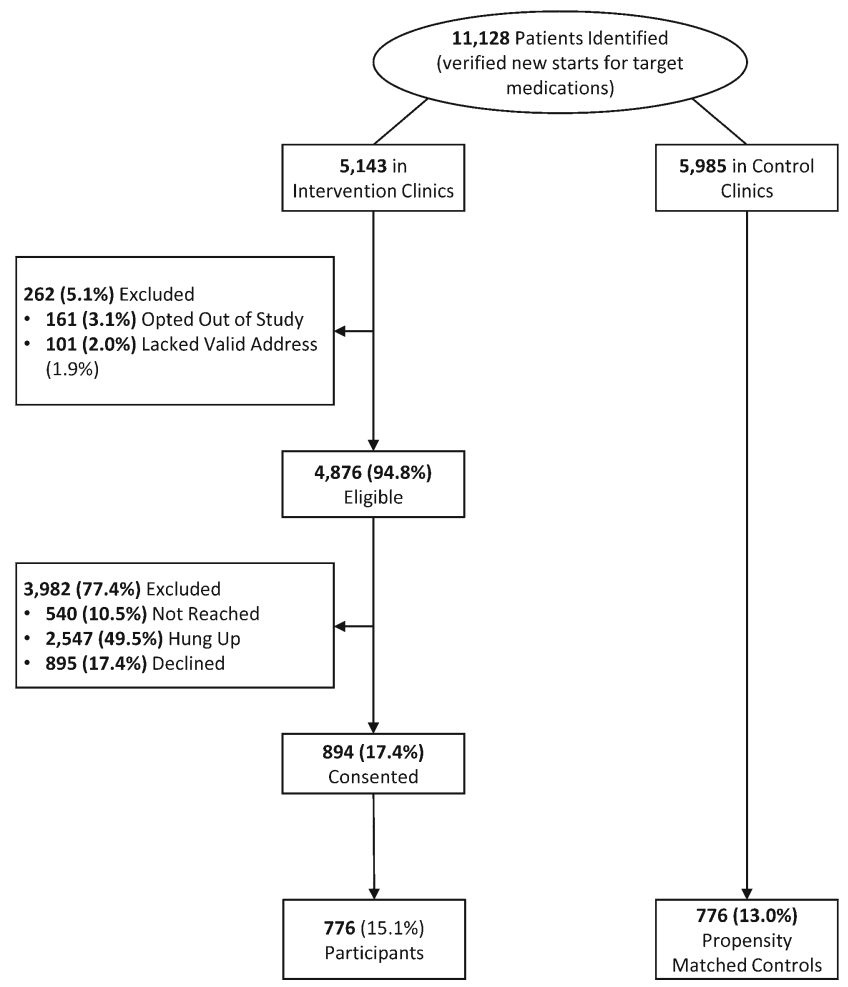

Figure 1 CONSORT study flow. Participants were defined as providing consent on the IVR call, completing birthdate verification, and inputting at least one question on the IVR survey. medication; $15.7 \%$ were taking a medication to treat depression; and $12.9 \%$ were prescribed an oral medication to treat diabetes.

\section{Automated (IVR) Survey and Pharmacist Assessment}

Of the 776 participants, 643 (82.9\% of IVR participants) completed all relevant questions in the IVR survey; 583 (75.1\%) met the requirements for automatic transfer to the pharmacist, either because they reported a potential side effect since starting the target medication (414 participants; $53.4 \%$ ) or because they requested to speak to a pharmacist (169 patients; $21.8 \%$ ). Three hundred twenty $(41.2 \%)$ patients successfully transferred to the pharmacist, and slightly fewer $(313 ; 40.3 \%)$ completed a phone-based encounter with the pharmacist. The remaining 263 calls eligible for transfer were either terminated or the patient declined the live encounter. The pharmacist talked about symptoms with 285 (36.7\%) patients and other health-related topics with $28(3.6 \%)$ patients (Fig. 2).

The 776 participants reported 997 total new potentially drug-related symptoms to the IVR system; many of these were subsequently discussed with the pharmacist by the subset of patients who had their call transferred and stayed on the line. The 285 patients who spoke to the pharmacist in real-time discussed 1018 potential ADE-related symptoms. These patient-reported symptoms were related to 52 different medications. Of these, 188 (18.5\%) were assessed as probably related and 479 (47.1\%) possibly related to the newly initiated medication. Of the symptoms assessed as probably or possibly medication-related, 266 $(39.8 \%)$ were rated as mild, $400(59.9 \%)$ as significant, and $1(0.15 \%$, suicidal ideation) as life-threatening using previously validated criteria (Table 2). ${ }^{20,21}$ Review of the EHR found that physicians explicitly referenced the pharmacist conversation/documentation in their own notes in 23 patient encounters $(8.1 \%$ of those with a pharmacist note documenting patient symptoms).

\section{Documentation of Symptoms and Drug Discontinuations}

For our primary measure comparing physician documentation of patient-reported drug-related symptoms, we compared IVR participants with a propensity-matched cohort of control patients and found significant increases in documentation of symptoms, number of symptoms documented, and number of patients with symptoms documented in the intervention group. Detailed review of these clinicians' notes identified a total of 277 symptoms that they specifically documented as potentially related to the index medication, compared to 164 in the propensity-matched control patients ( $p<0.0001$, Table 2$)$. We observed a greater number of unique symptoms documented and unique patients with symptoms documented in the intervention group ( $p<0.0001$ for each category). 


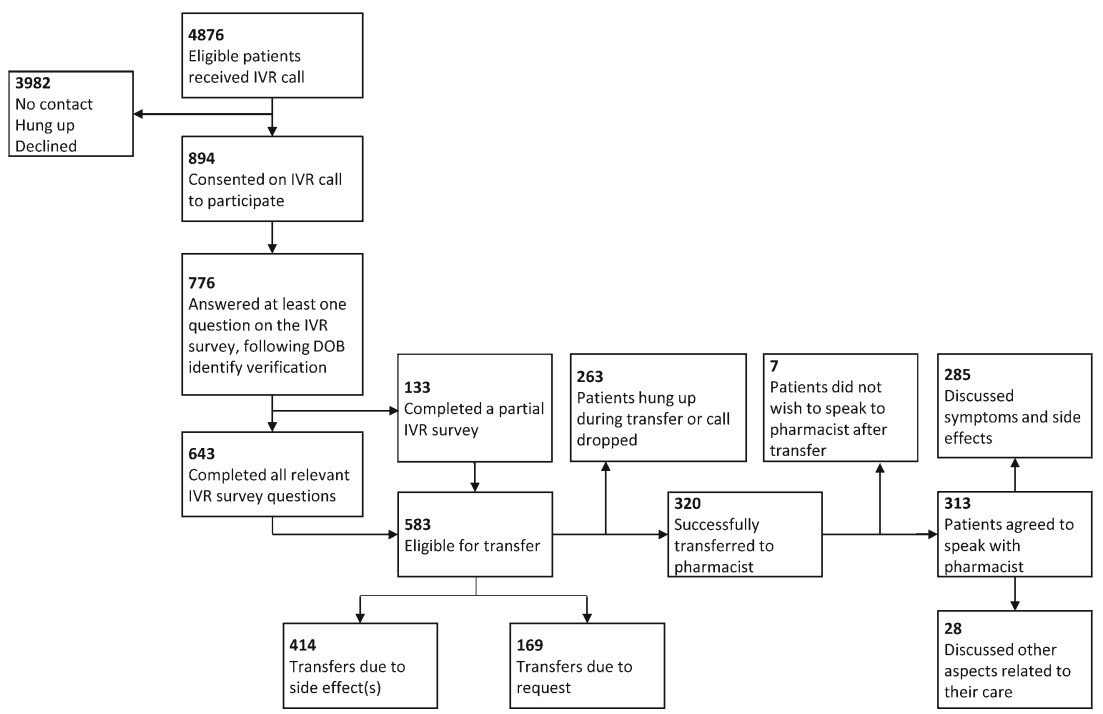

Figure 2 Uptake of IVR and pharmacist counseling intervention.

Analyzing the results by overall intention to treat (entire cohort assigned to intervention vs. control) using our EMRbased discontinuation rates with structured reasons, there was no significant difference in medications discontinued; however, there was a highly significant increased proportion discontinued due to adverse events in the intervention group (15\% vs. $11 \%, p=0.0003$ ) (Table 2).

Table 3 summarizes frequencies of symptoms that patients reported on the IVR calls. It shows the frequencies of symptoms by each of the four conditions being treated and reveals that patients being treated with medications for depression and insomnia reported more symptoms overall.

\section{DISCUSSION}

Patients starting medications frequently experience drugrelated symptoms that may be unreported or overlooked. We deployed an IVR pharmacosurveillance system coupled with pharmacist transfer and uncovered a significant number of

Table 2 Documented Symptoms and Drug Discontinuations, Intervention vs. Control

\begin{tabular}{|c|c|c|c|}
\hline & $\begin{array}{l}\text { Intervention participants } \\
n=776\end{array}$ & $\begin{array}{l}\text { Control patients } \\
n=776\end{array}$ & $p$ value \\
\hline Total symptoms collected by IVR system & 997 & NA & \\
\hline Total symptoms discussed by pharmacist & 1018 & NA & \\
\hline Related to target medication - possible & $479(47.1 \%)$ & NA & \\
\hline Related to target medication-unlikely & $351(34.5 \%)$ & NA & \\
\hline \multicolumn{4}{|l|}{$\begin{array}{l}\text { Severity of pharmacist-confirmed possible and probable } \\
\text { symptoms }(N=668)\end{array}$} \\
\hline Life-threatening & $1(0.15 \%)$ & NA & \\
\hline Total symptoms documented by MD in notes & 277 & 164 & $<0.0001$ \\
\hline Number of symptoms per 100 patients & 36 & 21 & $<0.0001$ \\
\hline Number of patients with symptoms documented by MD & 177 & 122 & $<0.0001$ \\
\hline $\begin{array}{l}\text { Total unique symptoms documented (including IVR } \\
\text { documented and MD notes) }\end{array}$ & 1303 & 164 & $<0.0001$ \\
\hline $\begin{array}{l}\text { Total unique patients with symptoms documented } \\
\text { (including IVR documented) }\end{array}$ & 448 & 120 & $<0.0001$ \\
\hline Drug discontinuations (CPOE $\mathrm{d} / \mathrm{c}$ code reason) & $\begin{array}{l}N=5106^{*} \\
\text { No. }(\%)\end{array}$ & $\begin{array}{l}N=5897^{\dagger} \\
\text { No. }(\%)\end{array}$ & \\
\hline Discontinuations 1 year from prescription & $1694(33.2)$ & $1977(33.5)$ & 0.70 \\
\hline Discontinuations due to adverse event ${ }^{\ddagger}$ & $254(15.0)$ & $217(11.0)$ & 0.0003 \\
\hline
\end{tabular}

*Missing data on target medication discontinuations for 37 patients

†Missing data on target medication discontinuations for 88 patients

fDiscontinuation due to an adverse event as a coded justification in the EHR 
Table 3 Patient-Reported Symptoms on IVR

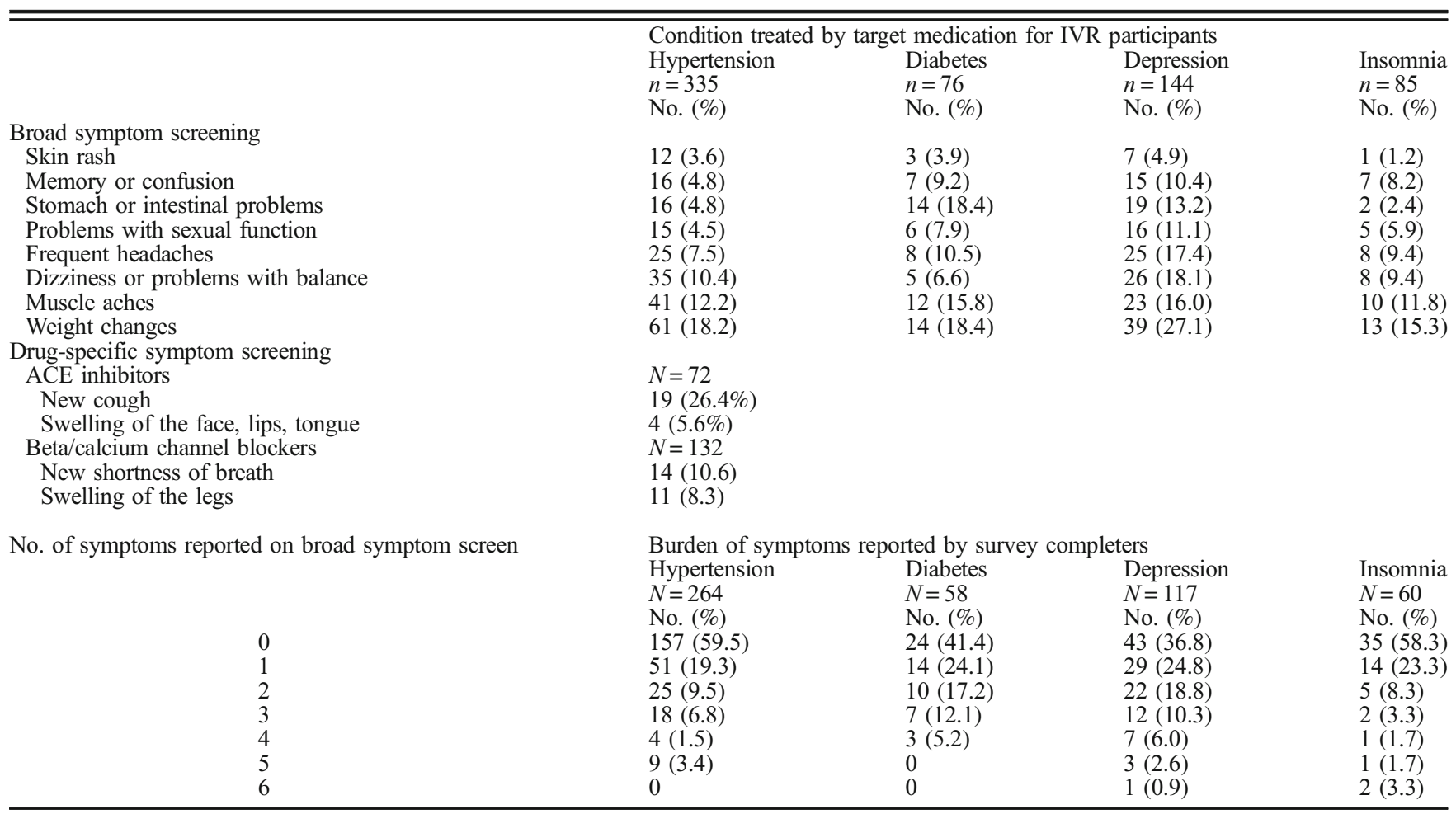

patient-reported symptoms, many of which were later validated as likely drug-related by pharmacist assessment and/or documentation in physician notes. Compared to a matched control group, there was a $50 \%$ increase in potential ADE symptoms documented by the PCP in patients who participated in the intervention compared to matched controls and when we also include symptoms reported to the IVR, a more than threefold increase (753 vs. 164) in total overall potential adverse symptoms documented. Despite the fact that only one of eight patients in the overall intervention group participated in the call, we nonetheless were able to observe a significant difference in the proportion of patients having their drugs discontinued by their physician with the physiciancoded discontinuation reason listed as an adverse event.

Reported symptoms varied by drug and target condition. Patients treated for insomnia and depression reported more symptoms on the IVR than patients newly started on drugs for diabetes or hypertension. These differences may be due either to patient- or medication-related factors. While patients reported a large number of non-specific symptoms on the IVR "since starting the (new) medication," the high rates also likely reflect an inherent bias that patients experiencing problems might be more likely to interact with our IVR and pharmacist outreach. Nonetheless, after careful review by our study pharmacist, nearly half met the criteria for being probably or possibly likely related to the newly started drugs.

It is important to note that many drug-related symptoms do not necessarily warrant discontinuation of the medication. A drug treatment's net risk and benefits must be weighed through shared decision-making between patient and prescriber, discussing various treatment options and alternatives (e.g., non-drug options, adjunctive medications, dosing alterations). Awareness of ADEs by the ordering clinician however facilitates such discussions and may also improve medication adherence. While heightening patients' awareness of possible adverse events might lead to greater non-adherence, our study pharmacist identified a number patients who were already not taking their medications as prescribed due to perceived symptoms, often unreported or unknown to their physicians.

Effectively and efficiently detecting ADEs is clinically and logistically challenging. ${ }^{22,} 23$ Prior studies have shown that manual phone-based outreach can identify symptoms, and automated calling systems such as IVR can facilitate largescale outreach $^{3,17,24,25}$ and, based on an economic analysis of this effort, can be done at reasonable cost. ${ }^{19}$ However, we encountered a number of challenges in implementing our IVR system that may limit broad application. In the past, patients typically had landline telephones and calls reached them at home at times they might be available to answer survey questions. Currently, many patients have mobile phones and have wearied of "robo-calls" from advertisers, politicians, and other surveys. ${ }^{26}$ As we documented in our suboptimal response rates and high percentage of patients who immediately hung up, the ability to reach patients using this technology has limitations on response rates. Because our calls came at unexpected and often inconvenient times, and patients 
might not have trusted that they were legitimately integrated into their healthcare, they may have had reservations about interacting with the system and providing confidential medical information.

Setting up and deploying an IVR system in a complex healthcare organization were also challenging. Although we created an algorithm to identify newly started medications, limitations in our system's coded records, new patient referrals, and complexities of our medication ordering process resulted in ongoing prescriptions initially being inaccurately labeled as new starts, requiring manual review to remove these ineligible cases. Creating the IVR script required extensive efforts and iterative testing with a professional vendor to ensure the logic and content of the call obtained accurate answers and was easy to use and interpret. Finally, the study's measurement was subject to limitations related to the fact that charts contained the pharmacist's notes; thus, reviewers could not be fully blinded to intervention vs. control patients (although we applied strict predefined criteria to the review solely of the physician notes), as well as any potential measured and unmeasured differences confounding our propensity-matched selected controls.

Despite these limitations, our automated system was able to collect a substantial number of previously unidentified drugrelated symptoms from more than $15 \%$ of a large cohort of patients. The IVR scripts we prepared can serve as ready-made templates for others seeking to do similar surveillance. Newer technologies such as text messaging, email communication, or patient portals could build on this work, overcoming some of the IVR hurdles we encountered and facilitating more convenient outreach. ${ }^{23,26,27}$ Regardless of what methods ultimately prove most efficient, this and other studies demonstrate that many ADEs are present and are often overlooked, yet could be detected with more systematic, proactive, and timely surveillance.

Acknowledgments: The team would like to acknowledge Japneet Kwatra, SM for her research assistance.

Corresponding Author: Gordon D. Schiff, MD; Division of General Internal Medicine and Primary Care Brigham and Women's Hospital, Boston, MA, USA (e-mail: gschiff@partners.org).

Funding This research was supported by the Agency for Healthcare Research and Quality (AHRQ), H.I.T Center for Education and Research on Therapeutics (CERT) grant number 1U19HS021094-01, part of AHRQ's CERT funding mechanism.

\section{Compliance with Ethical Standards:}

The research protocol was approved by the PHS Institutional Review Board and registered at Clinicaltrials.gov (NCT NCT02087293).

Disclaimer: The content is solely the responsibility of the authors and does not necessarily represent the official views of AHRQ.

Conflict of Interest: Dr. Schiff previously received grant compensation to evaluate the Medaware software. Dr. Bates consults for EarlySense, which makes patient safety monitoring systems. He receives cash compensation from CDI (Negev), Ltd., which is a not-for-profit incubator for health IT startups. He receives equity from ValeraHealth which makes software to help patients with chronic diseases. He receives equity from Clew which makes software to support clinical decisionmaking in intensive care. He receives equity from MDClone which takes clinical data and produces deidentified versions of it. Dr. Schiff's and Dr. Bates' financial interests have been reviewed by Brigham and Women's Hospital and Partners HealthCare in accordance with their institutional policies.

The remaining authors declare no potential conflicts of interest.

\section{REFERENCES}

1. CDC. National Center for Health Statistics: Therapeutic Drug Use. 2015; http://www.cdc.gov/nchs/fastats/drug-use-therapeutic.htm. Accessed 12/5/2016.

2. Kantor ED, Rehm CD, Haas JS, Chan AT, Giovannucci EL. Trends in prescription drug use among adults in the united states from 1999-2012. JAMA 2015;314(17):1818-1830.

3. Gandhi TK, Weingart SN, Borus $\mathbf{J}$, et al. Adverse drug events in ambulatory care. N Engl J Med 2003;348(16):1556-1564.

4. Taché SV, Sönnichsen A, Ashcroft DM. Prevalence of adverse drug events in ambulatory care: a systematic review. Ann Pharmacother 2011;45(7-8):977-989.

5. Budnitz DS, Shehab N, Kegler SR, Richards CL. Medication use leading to emergency department visits for adverse drug events in older adults. Ann Intern Med 2007;147(11):755-765.

6. Budnitz DS, Lovegrove MC, Shehab N, Richards CL. Emergency hospitalizations for adverse drug events in older Americans. N Engl J Med 2011;365(21):2002-2012.

7. Shehab N, Lovegrove MC, Geller AI, Rose KO, Weidle NJ, Budnitz DS. US emergency department visits for outpatient adverse drug events, 2013-2014. JAMA 2016;316(20):2115-2125.

8. Morrato EH. The Case for Funding Our National Drug Safety Surveillance System. In: LWW; 2017.

9. Classen DC, Resar R, Griffin F, et al. 'Global trigger tool'shows that adverse events in hospitals may be ten times greater than previously measured. Health Aff 2011;30(4):581-589.

10. Bourgeois FT, Shannon MW, Valim C, Mand1 KD. Adverse drug events in the outpatient setting: an 11-year national analysis. Pharmacoepidemiol Drug Saf 2010;19(9):901-910.

11. Weissman JS, Schneider EC, Weingart SN, et al. Comparing patientreported hospital adverse events with medical record review: do patients know something that hospitals do not? Ann Intern Med 2008;149(2):100108.

12. Berner ES, Ray MN, Panjamapirom A, et al. Exploration of an automated approach for receiving patient feedback after outpatient acute care visits. J Gen Intern Med 2014;29(8):1105-1112.

13. Berner ES, Burkhardt JH, Panjamapirom A, Ray MN. Cost implications of human and automated follow-up in ambulatory care. Am J Manag Care. 2014;20(11 Spec No. 17):SP531-540.

14. Willig JH, Krawitz M, Panjamapirom A, et al. Closing the feedback loop: an interactive voice response system to provide follow-up and feedback in primary care settings. J Med Syst 2013;37(2):9905.

15. Auger C, Forster AJ, Oake N, Tamblyn R. Usability of a computerised drug monitoring programme to detect adverse drug events and noncompliance in outpatient ambulatory care. BMJ Qual Saf 2013;22(4):306-316.

16. Houser SH, Ray MN, Maisiak R, et al. Telephone follow-up in primary care: can interactive voice response calls work? Stud Health Technol Inform 2013;192:112-116.

17. Haas JS, Iyer A, Orav EJ, Schiff GD, Bates DW. Participation in an ambulatory e-pharmacovigilance system. Pharmacoepidemiol Drug Saf 2010;19(9):961-969.

18. Haas JS, Amato M, Marinacci L, Orav EJ, Schiff GD, Bates DW. Do package inserts reflect symptoms experienced in practice?: assessment using an automated phone pharmacovigilance system with varenicline and zolpidem in a primary care setting. Drug Saf 2012;35(8):623-628.

19. Mahida S, Seoane E, Klinger E, et al. Cost of reporting possible adverse Drug reactions in medical Outpatients using a Telephonic interactive Voice response system. Value Health 2015;18(3):A257.

20. Rehan HS, Chopra D, Kakkar AK. Physician's guide to pharmacovigilance: Terminology and causality assessment. Eur $\mathrm{J}$ Intern Med 2009;20(1):3-8. 
21. Farcas A, Bojita M. Adverse drug reactions in clinical practice: a causality assessment of a case of drug-induced pancreatitis. J Gastrointestin Liver Dis 2009; 18(3):353-358.

22. Bates DW, Evans RS, Murff H, Stetson PD, Pizziferri L, Hripcsak G. Detecting adverse events using information technology. J Am Med Inform Assoc 2003;10(2): 115-128.

23. Forster AJ, Erlanger TE, Jennings A, et al. Effectiveness of a computerized drug-monitoring program to detect and prevent adverse drug events and medication non-adherence in outpatient ambulatory care: study protocol of a randomized controlled trial. Trials 2015;16(1):1

24. Forster AJ, Auger C. Using information technology to improve the monitoring of outpatient prescribing. JAMA Intern Med 2013;173(5):382-384
25. Falconi M, Johnston S, Hogg W. A scoping review to explore the suitability of interactive voice response to conduct automated performance measurement of the patient's experience in primary care. Prim Health Care Res Dev 2016;17(3):209-225.

26. Boulos MNK, Wheeler S, Tavares C, Jones R. How smartphones are changing the face of mobile and participatory healthcare: an overview, with example from eCAALYX. Biomed Eng Online 2011;10(1):1.

27. Link MW, Murphy J, Schober MF, et al. Mobile technologies for conducting, augmenting and potentially replacing surveys: report of the AAPOR task force on emerging technologies in public opinion research. Deerfield: AAPOR 2014. 